\title{
ZEROES OF THE COHOMOLOGY OF THE STEENROD ALGEBRA
}

\author{
ARUNAS LIULEVICIUS ${ }^{1}$
}

1. Introduction. The paper is devoted to proving the analogue of the Adams' vanishing theorem [4] for the cohomology of the Steenrod algebra $A$ over $Z_{p}$, where $p$ is an odd prime. The result is used to obtain a better bound on the order of elements in the stable homotopy groups of spheres. The methods of proof are analogues of [4].

Let $A_{0}$ be the subalgebra of $A$ consisting of 1 and $Q_{0}$ [9]; $A_{0}$ has a natural $A$-module structure consistent with the inclusion $A_{0} \subset A$.

Theorem 1. Let $M$ be any $A_{0}$-free $A$-module such that $M_{t}=0$ for $t<m$. Then $\operatorname{Ext}_{A}^{t_{i} t}\left(M, Z_{p}\right)=0$ for $t<m+(2 p-1) s-1, s \geqq 1$.

Corollary 1. $\operatorname{Ext}_{A}^{s, t}\left(Z_{p}, Z_{p}\right)=0$ for $t<(2 p-1) s-2, s \geqq 1$.

THEOREM 2. Let $\Pi_{r}^{S}$ be the rth stable homotopy group of the sphere, $p$ an odd prime. Then $\Pi_{r}^{S}$ contains no p-elements of order $>p^{[(r+2) / 2(p-1)]}$.

2. Preliminary computations. Let $A$ be the Steenrod algebra [9] over $Z_{p}, p$ an odd prime. Let $A_{r}$ be the subalgebra of $A$ generated by 1 and $Q_{0}, P^{p k}, k=0, \cdots, r-1$ (we set $P^{-1}=0, A_{\infty}=A$ ). Each $A_{r}$ is a Hopf subalgebra of $A_{8}, s \geqq r$, therefore [10] $A_{8}$ is free as a left (or right) $A_{r}$-module. The subalgebra $A_{0}$ is a left $A_{r}$-module, the module structure being consistent with the inclusion $A_{0} \subset A_{r}$.

Proposition 1. If $s \geqq r$, then $A_{s} \otimes_{A_{r}} A_{0}$ is free as a left $A_{0}$-module.

Proof. Consider the graded dual $A_{s}^{*}$ of $A_{s}$ :

$$
A_{s}^{*}=\Lambda_{p}\left[\tau_{0}, \cdots, \tau_{s}\right] \otimes z_{p} Z_{p}\left[\xi_{1}, \cdots, \xi_{r}\right] / I_{r},
$$

where $I_{r}$ is the ideal in the polynomial ring generated by $\xi_{1}^{p^{r}}, \cdots$, $\xi_{k}^{p^{r k+1}}, \cdots, \xi_{r}$ (see [9]). The proof is completed by exhibiting $\left(A_{\mathrm{s}} \otimes_{A_{\mathrm{r}}} A_{0}\right)^{*}$ as a subspace of $A_{\mathrm{s}}{ }^{*} \otimes A_{0}{ }^{*}$, and proving that the former is a free left $A_{0}{ }^{*}$-comodule. For this purpose it is convenient to replace $\tau_{i}$ and $\xi_{j}$ in (1) by $c\left(\tau_{i}\right), c\left(\xi_{j}\right)$, where $c$ is the conjugation antiautomorphism.

We wish to study the groups $\operatorname{Ext}_{A}\left(Z_{p}, Z_{p}\right)$. Let us write $\beta \in(s, t)$ if $\beta \in \operatorname{Ext}_{A}^{s, t}\left(Z_{p}, Z_{p}\right)$. These groups have been computed completely

Received by the editors July 15, 1962.

1 The author holds a National Science Foundation postdoctoral fellowship. 
in [5] for $t-s \leqq 2 p(p-1)-1$. The results are as follows: there are classes

$$
\begin{gathered}
1 \in(0,0), \quad \alpha_{0} \in(1,1), \quad h_{i} \in\left(1,2 p^{i}(p-1)\right), \\
\lambda_{i} \in\left(2,2 p^{i+1}(p-1)\right), \quad \rho_{s} \in(s, 2 s p-s-1), \quad 2 \leqq s \leqq p,
\end{gathered}
$$

such that the following elements constitute $Z_{p}$-bases for $(s, *)$ in total degrees $t-s \leqq 2 p(p-1)-1$ :

$$
\begin{aligned}
& 1 \text { for }(0, *) \alpha_{0}, h_{0}, h_{1} \text { for }(1, *) \\
& \alpha_{0}^{*}, \rho_{s}, \alpha_{0}^{8-2} \lambda_{0}, \alpha_{0}^{*-1} h_{1} \text { for }(s, *), \quad 2 \leqq s \leqq p-1, \\
& \alpha_{0}^{*} \text { for }(s, *) \quad \text { with } s>p .
\end{aligned}
$$

The elements (2) also satisfy the relations

$$
\alpha_{0} h_{0}=0, \quad \alpha_{0} \rho_{s}=0, \quad \alpha_{0}^{p-1} \lambda_{0}=0, \quad \alpha_{0}^{p} h_{1}=0 .
$$

The information in (2)-(4) allows us to compute a good part of $\operatorname{Ext}_{A}\left(A_{0}, Z_{p}\right)$ (see [1]). In particular, we have

LEMMA 1. $\operatorname{Ext}_{A}^{s, t}\left(A_{0}, Z_{p}\right)=0$ for $1 \leqq s \leqq p, t<2 p s-s-1$.

Proposition 2. If $M$ is an $A$-module which is $A_{0}$-free and $M_{t}=0$ for $t<m$, then $\operatorname{Ext}_{A}^{s, t}\left(M, Z_{p}\right)=0$ for $1 \leqq s \leqq p, t<m+2 p s-s-1$.

Proof. In the spirit of Lemma 3 of [3]. Induction and Five Lemma.

Proposition 3. Suppose that for any $M$ as in Proposition 2 we have $\operatorname{Ext}_{A}^{s, t}\left(M, Z_{p}\right)=0$ for $t<m+F(s)$ for $s=1, \cdots, k$, then $\operatorname{Ext}_{A}^{s+i, t}\left(M, Z_{p}\right)$ $=0$ for $t<m+F(k)+F(i), i=1, \cdots, k$.

Proof. Consider a minimal resolution [2] of $M$ as an $A$-module. Let $N$ be the module of $(k-1)$-cycles. Then $N_{t}=0$ for $t<m+F(k)$. Since $A$ and $M$ are $A_{0}$-free, so is $N$. Applying the hypothesis of the proposition to $N$, we have $\operatorname{Ext}_{A}^{i, t}\left(N, Z_{p}\right)=0$ for $t<m+F(k)+F(i)$, $i=1, \cdots, k$. The proof is completed by remarking that

$$
\operatorname{Ext}_{A}^{i, t}\left(N, Z_{p}\right) \cong \operatorname{Ext}_{A}^{k+i, t}\left(M, Z_{p}\right) \text {. }
$$

Corollary. If $M$ is any $A_{0}$-free $A$-module with $M_{t}=0$ for $t<m$, then $\operatorname{Ext}_{A}^{s, t}\left(M, Z_{p}\right)=0$ for $t<m+T(s)$, where $s>0$ and $T(r p+j)$ $=r\left(2 p^{2}-p-1\right)+2 p j-j-1, j=1, \cdots, p$.

Proposition 4. Let $i: A_{r} \rightarrow A$ be the inclusion map. Under the hypotheses of Proposition 3 (with $F(s) \geqq F(s-1)$ ) 


$$
i^{*}: \operatorname{Ext}_{A}^{s, t}\left(M, Z_{p}\right) \rightarrow \operatorname{Ext}_{A_{r}}^{s, t}\left(M, Z_{p}\right)
$$

is an isomorphism for $t<m+F(s-1)+2 p^{r+1}(p-1)$, where $s=1, \cdots, k$.

Proof. Consider

$$
0 \rightarrow K \rightarrow A \otimes_{A_{r}} M \rightarrow M \rightarrow 0
$$

since $M$ and $A \otimes_{A_{r}} M$ are both $A_{0}$-free (Proposition 1), so is $K$. Also $K_{t}=0$ for $t<m+2 p^{r+1}(p-1)$. Thus $\operatorname{Ext}_{A}^{s, t}\left(K, Z_{p}\right)=0$ for $t<m$ $+2 p^{r+1}(p-1)+F(s)$. Since $F(s) \geqq F(s-1)$ (a trivial assumption), the proposition follows from the remark that

$$
\operatorname{Ext}_{A}^{s, t}\left(A \otimes_{A_{r}} M ; Z_{p}\right) \cong \operatorname{Ext}_{A_{r}}^{s, t}\left(M, Z_{p}\right),
$$

for $A$ is free as a right $A_{r}$-module [10].

3. The cohomology of $A_{1}$. We wish to compute $\operatorname{Ext}_{A_{1}}\left(Z_{p}, Z_{p}\right)$; we shall use the method of [8]. Let $Q_{1}=\left[P^{1}, Q_{0}\right]$ (see [9]); then we have the following relations:

$Q_{0} Q_{0}=0, \quad Q_{1} Q_{1}=0, \quad\left[Q_{0}, Q_{1}\right]=0, \quad\left(P^{1}\right)^{p}=0, \quad\left[P^{1}, Q_{1}\right]=0$.

Let $D$ be the exterior algebra generated by $Q_{1} ; D$ is a normal [10] Hopf subalgebra of $A_{1}$, and $G=A_{1} / / D$ is a tensor product of an exterior algebra on $e=Q_{0}+A_{1} \bar{D}$, and a truncated polynomial algebra on $a=P^{1}+A_{1} \bar{D}$. It is well known that

$$
\operatorname{Ext}_{a}^{*, *}\left(Z_{p}, Z_{p}\right)=Z_{p}[\alpha] \otimes \Lambda_{p}[\mu] \otimes Z_{p}[\lambda],
$$

where $\alpha \in(1,1), \mu \in(1,2 p-2), \lambda \in(2,2 p(p-1))$; similarly,

$$
\operatorname{Ext}_{D}^{*, *}\left(Z_{p}, Z_{p}\right)=Z_{p}[\beta]
$$

where $\beta \in(1,2 p-1)$.

We can describe minimal resolutions for $Z_{p}$ over $G$ and $D$ very easily: for the minimal resolution $Y=G \otimes \bar{Y}$ we take a complex with $G$-free generators $\left[\alpha^{k} \mu^{\epsilon} \lambda^{m}\right]$, where $\epsilon=0,1, k, m=0,1,2, \cdots$, and define the differential $d^{\prime}$ as follows:

$$
\begin{aligned}
d^{\prime}\left[\alpha^{k} \mu \lambda^{m}\right] & =e\left[\alpha^{k-1} \mu \lambda^{m}\right]+a\left[\alpha^{k} \lambda^{m}\right], \\
d^{\prime}\left[\alpha^{k} \lambda^{m+1}\right] & =e\left[\alpha^{k-1} \lambda^{m+1}\right]+a^{p-1}\left[\alpha^{k} \mu \lambda^{m}\right] .
\end{aligned}
$$

Similarly, for the minimal resolution $W=D \otimes \bar{W}$ we take a complex with $D$-free generators $\left[\beta^{r}\right], r=0,1,2, \cdots$, and differential $d^{\prime \prime}$ :

$$
d^{\prime \prime}\left[\beta^{r}\right]=Q_{1}\left[\beta^{r-1}\right] \text {. }
$$

REMARK. The reader is cautioned that we are using the (reasonable) sign convention: any time two maps (with degree and grading) 
are switched past each other, we multiply by -1 raised to the product of total degrees. Example: a $D$-map $f$ of degree 1 and grading 0 satisfies $f\left(Q_{1} m\right)=-Q_{1} f(m)$.

We construct an $A_{1}$-resolution of $Z_{p}$ by introducing a suitable differential $d$ in $A_{1} \otimes \bar{Y} \otimes \bar{W}$ (compare [8]). We let $d=\sum_{k=0}^{\infty} d_{k}$, where $d_{0}$ is induced by $d^{\prime \prime}$ :

$$
d_{0}\left[\alpha^{k} \mu^{e} \lambda^{m}\right] \otimes\left[\beta^{r}\right]=(-1)^{\complement}\left[\alpha^{k} \mu \lambda^{m}\right] \otimes Q_{1}\left[\beta^{r-1}\right]=Q_{1}\left[\alpha^{k} \mu^{\epsilon} \lambda^{m}\right] \otimes\left[\beta^{r-1}\right],
$$

and $d_{k}, k \geqq 1$, are defined as follows:

$$
\begin{aligned}
& d_{1}\left(\left[\alpha^{k} \mu \lambda^{m}\right] \otimes\left[\beta^{r}\right]\right)=e\left[\alpha^{k-1} \mu \lambda^{m}\right] \otimes\left[\beta^{r}\right]+a\left[\alpha^{k} \lambda^{m}\right] \otimes\left[\beta^{r}\right], \\
& d_{1}\left(\left[\alpha^{k} \lambda^{m+1}\right] \otimes\left[\beta^{r}\right]\right)=e\left[\alpha^{k} \lambda^{m+1}\right] \otimes\left[\beta^{r}\right]+a^{p-1}\left[\alpha^{k} \mu \lambda^{m}\right] \otimes\left[\beta^{r}\right], \\
& d_{2}\left(\left[\alpha^{k} \mu \lambda^{m}\right] \otimes\left[\beta^{r}\right]\right)=-(r+1)\left[\alpha^{k-1} \lambda^{m}\right] \otimes\left[\beta^{r+1}\right], \\
& d_{q}\left(\left[\alpha^{k} \mu \lambda^{m}\right] \otimes\left[\beta^{r}\right]\right)=0, \quad q \geqq 3, \\
& d_{j}\left(\left[\alpha^{k} \lambda^{m+1}\right] \otimes\left[\beta^{r}\right]\right)=(j-1) !\left(\begin{array}{c}
r+j-1 \\
j-1
\end{array}\right) a^{p-j}\left[\alpha^{k-j+1} \mu \lambda^{m}\right] \otimes\left[\beta^{r+j-1}\right], \\
& \text { for } j \geqq 2 .
\end{aligned}
$$

Since $d_{k}, k=0,1, \cdots$, satisfy the conditions of Theorem 1 of [8], $A_{1} \otimes \bar{Y} \otimes \bar{W}$ yields an $A_{1}$-resolution of $Z_{p}$.

The elements $[\alpha],[\lambda],[1] \otimes\left[\beta^{p}\right],[\mu] \otimes\left[\beta^{j}\right], j=0,1, \cdots, p-2$ yield elements in $\operatorname{Tor}_{*, *}^{A_{1}}\left(Z_{p}, Z_{p}\right)$. Denote by $\alpha, \lambda, \omega, \rho_{j+1}, j=0,1$, $\cdots, p-2$, their duals in $\operatorname{Ext}_{A_{1}}^{*, *}\left(Z_{p}, Z_{p}\right)$. We then immediately have:

Proposition 5. $\operatorname{Ext}_{A_{1}}\left(Z_{p}, Z_{p}\right)$ is a free $Z_{p}[\omega]$-module with a set of free generators given by the elements

$$
\alpha^{k}, \quad \alpha^{i} \lambda^{m}, \quad \rho_{8} \lambda^{m},
$$

where $k=0,1,2, \cdots, j=0,1, \cdots, p-2, s=1, \cdots, p-1$. The elements satisfy the relations

$$
\alpha \rho_{s}=0, \quad \alpha^{p-1} \lambda=0 .
$$

The next proposition is now trivial.

Proposition 6. (i) $\operatorname{Ext}_{A_{1}}^{s, t}\left(A_{0}, Z_{p}\right)=0$ if $t<(2 p-1) s-1, s>0$;

(ii) multiplication by $\omega$ is an isomorphism in $\operatorname{Ext}_{A_{1}}^{s, t}\left(A_{0}, Z_{p}\right)$ for $t<(2 p-1) s+2 p^{2}-6 p+1$.

Corollary. If $M$ is an $A_{1}$-module which is $A_{0}$-free, and $M_{t}=0$ for $t<m$, then $\operatorname{Ext}_{A_{1}}^{s, t}\left(M, Z_{p}\right)=0$ if $t<m+(2 p-1) s-1$ and $s>0$.

Remark. We cannot prove Proposition 6 (ii) for general $A_{0}$-free $M$. However, it seems to be true for $M=\bar{A} / A \bar{A}_{0}$ : that is, $\operatorname{Ext}_{A}^{s, t}\left(Z_{p}, Z_{p}\right)$ seems to be periodic in a small neighborhood of the line $t=(2 p-1) s$ -2 . 
4. Proof of Theorems 1 and 2. Write $s=r p+i, i=1, \cdots, p$. Theorem 1 is proved by induction on $r$. For $r=0$ this is Proposition 2. We suppose that the theorem has been proved for $r^{\prime}<r$ and all $A_{0}$-free $M$; we then estimate the zeroes in dimensions $r p+i$ by using Proposition 3. Here Proposition 4 gives an isomorphism with $\operatorname{Ext}_{A_{1}}^{s, t}\left(M, Z_{p}\right)$ in a neighborhood of the line $t=m+(2 p-1) s-2$. According to the corollary of Proposition 6, this enables us to prove that $\operatorname{Ext}_{A}^{s, t}\left(M, Z_{p}\right)$ $=0$ for $t<m+(2 p-1) s-1$ for $s=r p+i, i=1, \cdots, p$, which completes the inductive step.

Corollary 1 follows from the observation that $N=\bar{A} / A \bar{A}_{0}$ is $A_{0^{-}}$ free and $N_{t}=0$ for $t<2 p-2$.

Proposition 6(ii) and Proposition 4 with $F(s)=(2 p-1) s-1$ prove the following:

Corollary 2. $\operatorname{Ext}_{A}^{s, t}\left(A_{0}, Z_{p}\right) \cong \operatorname{Ext}_{A}^{s+p, t+2 p^{2}-p}\left(A_{0}, Z_{p}\right)$ for $t<(2 p-1) s$ $+2 p^{2}-6 p+1$

Theorem 2 is an immediate consequence of Corollary 1 and the Adams spectral sequence [1].

REMARK. Theorem 2 shows that there are no elements of order $>p^{p^{k}}$ in dimension $r=2 p^{k}(p-1)-1$. Since the $\bmod p$ Hopf invariant is trivial for $k>0$ [7], there are no elements of order $>p^{p^{k}-1}$ in these dimensions. Theorem 2 should be compared with Theorem 7 of [6].

\section{REFERENCES}

1. J. F. Adams, On the structure and applications of the Steenrod algebra, Comment. Math. Helv. 32 (1958), 180-214.

2. - On the non-existence of elements of Hopf invariant one, Ann. of Math. (2) 72 (1960), 20-104.

3. - A finiteness theorem in homological algebra, Proc. Cambridge Philos. Soc. 57 (1961), 31-36.

4. - Stable homotopy theory (lecture notes), Berkeley, Calif., 1961.

5. H. Gershenson, Some relationships between the Adams spectral sequence and Toda's calculations of the stable homotopy of spheres, Dissertation, Univ. of Chicago, Chicago, Ill., 1961.

6. I. M. James, On the homotopy groups of spheres, Symposium Internacional de Topologia Algebraica, Mexico, 1958, 222-224.

7. A. Liulevicius, The factorization of cyclic reduced powers by secondary cohomology operations, Mem. Amer. Math. Soc. 42 (1962), 113 pp.

8. - The cohomology of a subalgebra of the Steenrod algebra, Trans. Amer. Math. Soc. 104 (1962), 443-449.

9. J. Milnor, The Steenrod algebra and its dual, Ann. of Math. (2) 67 (1958), 150-171.

10. J. Milnor and J. C. Moore, On the structure of Hopf algebras (to appear).

The University of Chicago and

The Institute for Advanced Study 